\title{
Using the Road Traffic Simulation "SUMO" for educational Purposes
}

\author{
Daniel Krajzewicz ${ }^{1}$, Markus Hartinger ${ }^{1}$, Georg Hertkorn ${ }^{1}$, Peter Mieth ${ }^{1}$, \\ Christian Rössel ${ }^{1}$, Julia Zimmer ${ }^{1}$, Peter Wagner ${ }^{1}$ \\ ${ }^{1}$ German Aerospace Centre, Institute for Transportation Research \\ Rutherfordstr. 2 - 12489 Berlin - Germany \\ email: Daniel.Krajzewicz@dlr.de
}

\begin{abstract}
Summary.
Since the year 2000, the Centre of Applied Informatics and the Institute for Transport Research at the German Aerospace Centre develops a microscopic road traffic simulation package named "SUMO" - an acronym for "Simulation of Urban MObility". Meanwhile, the simulation is capable to deal with realistic scenarios such as large cities and is used for these purposes within the institute's projects. The idea was to support the traffic research community with a common platform to test new ideas and models without the need to reimplement a framework that handles road data, vehicle routes, traffic light steering etc. To achieve this goal, the simulation code is available as open source.

Within this publication, we would like to demonstrate how most attributes of traffic flow can be simulated. This should be mainly interesting for educational purposes.

Keywords: traffic simulation, road traffic, car following, microscopic, continuous, multi-modal, open source, car-driver model, traffic research, education
\end{abstract}

\section{Introduction}

SUMO is a road traffic simulation package based on the microscopic carfollowing model developed by Stefan Krauß. A detailed description of this model's behaviour and fitting to real-world car behaviour can be found in [4], [7] and [8], a short description will follow.

SUMO by now consists of a set of applications, needed to perform the different steps of preparing a road traffic simulation. By now, the following modules exist: a network conversion utility, capable to import networks from other simulation packages such as Visum, Vissim or ARTEMIS but also from ArcView databases and from native XML descriptions, an OD-matrix to trip converter, a router (needed for traffic assignment) and two different versions of the simulation - one completely without a visualisation, used to perform fast simulations in a loop and a second one, slower, but with a graphical user interface written using openGL which is nevertheless surprisingly fast. We will not go into detail about the package itself, herein, as it has been described in some other places, such as [5]. 


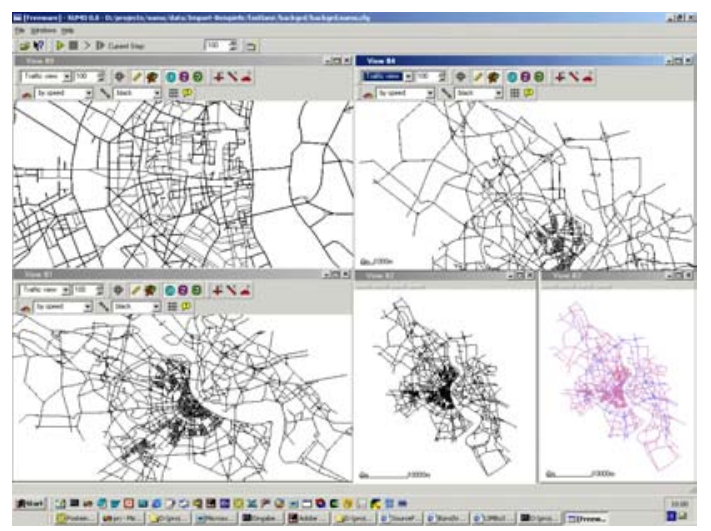

Fig. 1 A screenshot from a simulation of the city of Cologne, the Centre of Applied Information Science is located at, showing different zooming scales and colouring schemes (down right: streets coloured by the maximum speed allowed)

\section{The model}

The model developed by Krauß in 1998 is a microscopic, space-continuous, car-following model based on the safe speed - paradigm: a driver tries to stay away from the driver in his front at a distance and a safe speed that allows him to adapt this leader's deceleration. The model assumes the driver to have a reaction time tau of about one second. The model uses the following parameter:

a: $\quad$ the maximum acceleration of the vehicle $\left(\right.$ in $\left.\mathrm{m}^{\wedge} 2\right)$

b: the maximum deceleration of the vehicle (in (in $\mathrm{m} / \mathrm{s}^{\wedge} 2$ )

vmax: the maximum velocity of the vehicle (in $\mathrm{m} / \mathrm{s}$ )

1: $\quad$ the length of the vehicle (in $\mathrm{m}$ )

e: the driver's imperfection in holding the wished speed (between 0 and 1)

This safe velocity is computed using the following equation:

$v_{s a f e}(t)=v_{l}(t)+\frac{g(t)-v_{l}(t) \tau}{\frac{\bar{v}}{b(\bar{v})}+\tau}$

Where:

vl( $t)$ : speed of the leading vehicle in time $t$

$g(t): \quad$ gap to the leading vehicle in time $t$

tau: the driver's reaction time (usually $1 \mathrm{~s}$ )

As vsafe may be larger than the maximum speed allowed on the road he uses or larger than the vehicle is capable to reach until the next step due to his acceleration capabilities, the minimum of these values is computed as next. The resulting speed is called the "desired" or "wished" speed.

$v_{d e s}(t)=\min \left\{v_{s a f e}(t), v(t-1)+a, v_{\max }\right\}$

Assuming the driver is not able to perfectly adapt the desired velocity, the "driver's imperfection" value multiplied with the car's acceleration ability and a random number is substracted from the desired velocity. Finally, one 
must assure, the vehicle is not driving backwards. Due to this, the last of the model's equation is:

$v(t)=\max \left\{0, \operatorname{rand}\left[v_{\text {des }}(t)-\epsilon a, v_{\text {des }}(t)\right]\right\}$

The velocity, multiplied with the simulation step duration, which is constantly equal to one second, here, is added to the vehicle's current position to achieve the position for the next time step. This model is collision-free and due to the small number of equations very fast - on a $1 \mathrm{GHz}$ computer one can easily perform the simulation step for about 1.000 .000 vehicles in per second - each describing a real vehicle's movement within one second.

It is known within the traffic research that the traffic flow is a function of the traffic density. This is the fundamental diagram. As figure 2 shows, the model by Krauß is capable to replicate the flow function well.
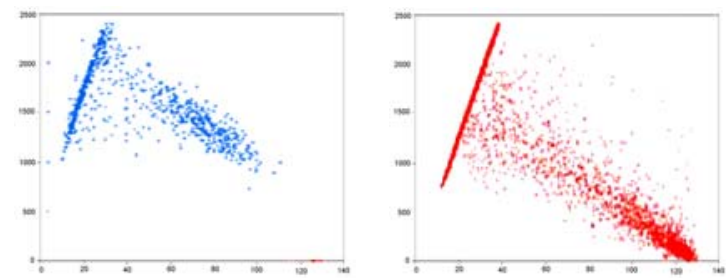

Fig. 2 The traffic flow as a function of the traffic density; left: original highway data, right: simulation results using the Krauß-model

\section{Possible traffic scenarios for educational purposes}

\subsection{Traffic assignment}

One important part of a traffic simulation of a large region is the dynamic assignment. In this process, the demand (O/D-matrix) is distributed onto the network. This is done by approaching a so-called dynamic user equilibrium (DUE), or in the case here, a stochastic dynamic UE (SDUE). This means that for any $\mathrm{O} / \mathrm{D}$-pair the flows on the various routes connecting $\mathrm{O}$ and $\mathrm{D}$ are distributed in such a manner, that the travel times (travel costs) are equal on all used routes.

This state is computed by an algorithm that has been described in Gawron [3]. SUMO uses this algorithm within its routing module. The simulation package includes two basic examples to demonstrate this approach. We will discuss only one here, which takes place within a network with only three streets. Driving from bottom to the top of the network, vehicles may use one of the side arms or the centre road. Within the first step, all vehicles use the centre road, as it seems to be the shortest one. Due to the arising high density and the fact, a traffic light is positioned at the road's end it's not. This is adapted by the drivers within the next iterations where each driver adapts the now fastest route with a certain probability. The plot within the next picture displays the overall number of simulated seconds until the last vehicle has left the simulated area. As one can see, drivers are gaining almost half of the time they needed if all use the path assumed to be the shortest one at the beginning. 


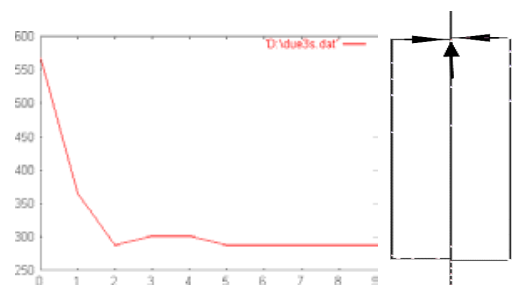

Fig. 3 The reduction of the simulation time until the last vehicle has ended his route over DUE-iterations as a measurement of the equilibrium-quality; left: the "network" used

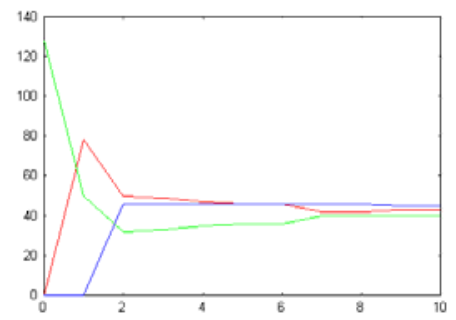

Fig. 4 Plot of the route usages over iterations; green: vehicles using the centre road, red: vehicles using the way to the right, blue vehicles using the way to the left

\subsection{Jam dynamics}

Jams are known to grow as long as the inflow stays high and wanders upwards to the stream. To visualise this behaviour, the following scenario was chosen: vehicles are emitted into a lane with a high density. A traffic light is placed at the end of this lane and with a very low frequency. This traffic light turns red causing a temporary jam. After it turns back green, one can see how the jam is moving backwards and grows. This scenario is included in the application's distribution, too. Still, the visualisation within a single plot is not done, yet.

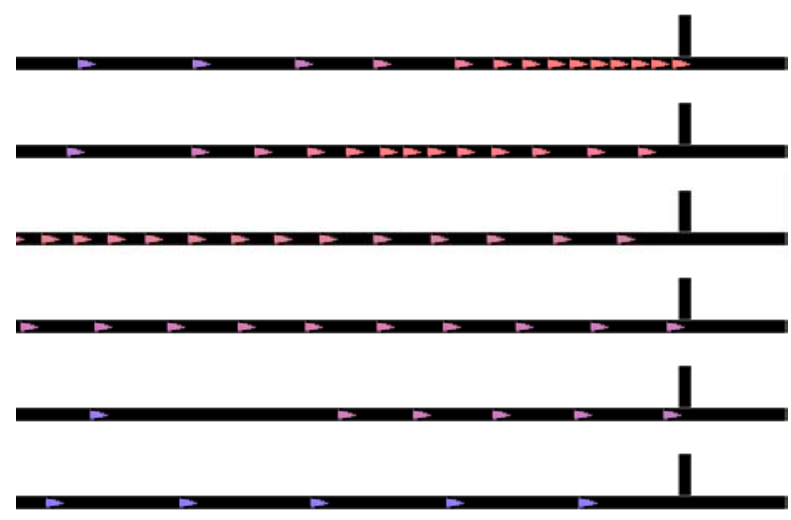

Fig. 5 The development of a jam - from the begin (top) back to free flow (bottom); the time difference between the pictures is $25 \mathrm{~s}$, the bluer a vehicle the faster it is, red vehicles are standing 


\subsection{Model calibration}

Investigations on microscopic models of traffic flow are one of the main research areas of the Institute of Transport Research and so many approaches are made to validate and calibrate these models (see [1] or [6]). It is assumed that drivers behave differently when different areas are regarded and so, the ability to being calibrated to a given set of traffic data is a traffic simulation's increasingly wished feature. The approach we use most often is to use realworld detector data and emit (release) vehicles on the begin of the road each time one of the real-world detectors was passed by a vehicle. Mostly, when using data from the I880-FSP for example, this is possible for each lane of a road.

In some distance, other real-world detector data are used to validate the simulation results. An external optimiser is then used to find a parameter set that fits best to the scenario. The next picture shows a preliminary result of the error development during the calibration process. A closer look will be given in another publication.

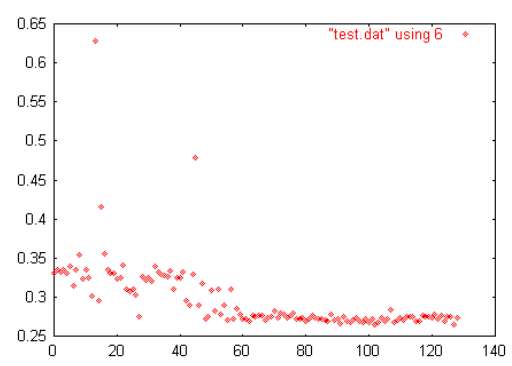

Fig. 5 The development of the error over simulation iterations

\section{Conclusion}

We hoped to be able to show that some prominent traffic predicates may be simulated with a small effort using the open source simulation package SUMO. Beside simple demonstrations as the ones described herein, the software is capable to deal with large scenarios and is already in use for such cases within the IVF. As open source software highly lives from his users, we hope so to encourage some of the readers to try it and share their experiences or ideas with us.

\section{References}

1. E. Brockfeld, R. Kühne, A. Skabardonis, P. Wagner. 2002. „Towards a Benchmarking of microscopic Traffic Flow Models“. TRB, in submission

2. E. W. Dijkstra. 1959. „A note on two problems in connection with graphs“. In: Numerische Mathematik, 1:269-271.

3. Christian Gawron. 1998. „Simulation-Based Traffic Assignment“. Inaugural Dissertation.

4. Stefan Janz. 1998. „Mikroskopische Minimalmodelle des Straßenverkehrs“. Diploma Thesis.

5. "SUMO (Simulation of Urban MObility); An open-Source Traffic Simulation". D Krajzewicz, G. Hertkorn, C. Rössel, P. Wagner. In: Proceedings of the 4th Middle East Symposium on Simulation and Modelling (MESM2002), Edited by: A. AlAkaidi, pp. 183 - 187, SCS European Publishing House, ISBN 90-77039-09-0 
6. D. Krajzewicz, G. Hertkorn, C. Rössel, P. Wagner; "An Example of Microscopic Car Models Validation using the Open Source Traffic Simulation SUMO"; In: Proceedings of Simulation in Industry, 14th European Simulation Symposium, 2002, Edited by: A. Verbraeck and W. Krug, pp. 318-322, SCS European Publishing House, ISBN 3-936150-21-4

7. Stefan Krauß. 1998. „Microscopic Modelling of Traffic Flow: Investigation of Collision Free Vehicle Dynamics"; Hauptabteilung Mobilität und Systemtechnik des DLR Köln. ISSN 1434-845

1. 8. Stefan Krauß, Peter Wagner, Christian Gawron. 1997. "Metastable States in a Microscopic Model of Traffic Flow"; Physical Review E, volume 55, number 304, pages 55-97; May, 1997 